\title{
Evaluation of push-out bond strength of different adhesive system applications
}

\author{
Emel Olga Önay \\ Department of Endodontics, Başkent University Faculty of Dentistry, Ankara, Turkey
}

\begin{abstract}
Objective: To compare interfacial strength and failure modes of glass-fibre endodontic posts luted with three different adhesive luting agents.

Methods: A total of 30 extracted human maxillary incisors were randomly divided in three groups and restored using glass-fibre posts and the following luting agents: an experimental self-etching primer/ resin-cement MSM-107 (EXP), ExciTE ${ }^{\circledR}$ F DSC adhesive/Variolink II (VII), Gradia Core self-etching bond/ Gradia Core cement (GC). Five sections of $1.00 \pm 0.05 \mathrm{~mm}$ thickness were prepared from each specimen, and the post in each section was subjected to a push-out test. Failure modes of root slices after push-out testing were examined with stereomicroscope. The level of significance was set at alpha=0.05.

Results: EXP achieved the highest bond strength. The mean value recorded for GC was significantly lower than EXP $(p<0.05)$ and did not differ from VII $(p>0.05)$. There was also no significant difference between EXP and VII ( $p>0.05$ ). Bond failure was mainly mixed failure for VII and GC. Adhesive failures between the cement and the post, cohesive failures within the post and the dentin were mostly observed in EXP.
\end{abstract}

Conclusion: The new experimental resin cement, MSM-107 showed promising results in term of bonding ability and might be used efficiently to restore endodontically treated teeth.

Keywords: Bonding agent; fiber post; luting cement; push-out bond strength.

F ibre-reinforced polymer posts have been employed to restore endodontically treated teeth that have suffered considerable loss of coronal structure since 1990s. ${ }^{[1,2]}$ They offer a number of advantages over custom-fabricated cast alloy posts and core or prefabricated alloy posts. Fibre posts more closely match the modulus of elasticity of sound root dentin, thus distributing occlusal stresses more evenly in the root dentin and providing higher fracture resistance to weakened roots. ${ }^{[3,4]}$ Other attractive features of fibre posts include resistance to corrosion, aesthetic appeal, and nonhypersensitivity. ${ }^{[5]}$
Selecting an appropriate adhesive and luting procedure for bonding posts to root dentine is challenging. Various luting agents and corresponding adhesive systems have been proposed for bonding fiber posts to root canal dentin. These materials can be divided into self-etching adhesives and etch \& rinse systems. ${ }^{[6]}$ Results on the effectiveness of self-etching adhesive systems when compared with etch \& rinses are contradictory. ${ }^{[7,8]}$ Some studies show similarities between these systems ${ }^{[8,9]}$ whilst others suggest a superiority of the etch \& rinse materials. ${ }^{[7,10]}$ In a confocal laser scanning microscopy study, the application

Correspondence: Dr. Emel Olga Önay. Başkent Üniversitesi Diş Hekimliği Fakültesi,

82. Sokak, No: 26, Bahçelievler, 06490 Ankara Turkey.

Tel: +90312 - 2030000 e-mail: olgaonay@yahoo.com

Submitted: March 28, 2016 Accepted: May 13, 2016 
of etch \& rinse and adhesives resulted in a higher number of resin tags and an increased hybrid layer thickness compared with self-etching adhesive systems. ${ }^{[1]}$

A new experimental etch \& rinse resin system (MSM107; GC, Tokyo, Japan) has been recently developed. However, no scientific information is so far available regarding its adhesive potential when used for fiber post luting.

The purpose of this study was to compare interfacial strength in the root canal amongst glass-fibre endodontic posts luted with different luting agents, using the pushout test method. The null hypothesis to be tested was that bond strengths to root canal dentin do not vary with type of luting system.

\section{Materials and methods}

A total of 30 intact human maxillary incisors with one straight root and, extracted because of periodontal reasons, were selected. Each tooth was placed in $5.25 \%$ sodium hypochlorite $(\mathrm{NaOCl})$ for $2 \mathrm{~h}$ for surface disinfection, and then stored in distilled water.

The crown of each tooth was sectioned at the cemento-enamel junction (CEJ) using a water-cooled diamond disk to obtain 14-mm long root. The working length was determined visually by subtracting $1 \mathrm{~mm}$ from the length of a size 10 file (K-Files Limas K; MANI, Tochigi, Japan) at the apical foramen. The middle and coronal thirds were prepared using 1, 2, 3, 4, 5 and 6 drills (Gates Glidden; Produits Dentaires, Vevey, Switzerland).

All canals were further prepared with R40 RECIPROC (40/0.06) file (VDW, Munich, Germany) as follows: The instrument was installed on a gear reduction handpiece powered by a torque-controlled motor (Gold; VDW $\mathrm{GmbH}$, Munich, Germany) and was introduced into the canals with back-and forth movements. After pecking for 3 times and when the blocking sensation was felt, the instrument was removed, cleaned and the canal was irrigated with $5 \% \mathrm{NaOCl}$ solution (NICLOR 5, Ogna, Maggio, Italy). This cycle was repeated until reaching the working length. Finally, the canals were rinsed with distilled water, dried with paper points and filled with sealer (Kerr Pulp Canal Sealer; Sybron Dental Specialties, CA, USA) and .06 taper gutta-percha points (RECIPROCGutta-Percha points; VDW GmbH, Munich, Germany) using the warm vertical condensation technique (BeeFill 2inl system; VDW GmbH, Munich, Germany). The filled roots were sealed with glass-ionomer cement (Fuji II; GC, Tokyo, Japan), and stored in water for $48 \mathrm{~h}$ to allow the sealer to set.

The post space was prepared with a low-speed drill provided by the post manufacturer up to a fixed depth of $10 \mathrm{~mm}$ from the CEJ. Following preparation, the canals were rinsed with $5 \% \mathrm{NaOCl}$ solution, followed with distilled water, and then canals were dried with paper points. The specimens were randomly divided into three groups of 10 teeth each. The translucent and radiopaque methacrylate-based glass-fiber posts (GC Fiber Posts, diameter $1.4 \mathrm{~mm}$; GC America Inc., Alsip, IL, USA) were luted with three different adhesive luting systems: An experimental self-etching primer/resin-cement MSM-107 (GC, Tokyo, Japan) (group 1), ExciTE ${ }^{\circledR}$ F DSC adhesive/ Variolink II (Ivoclar Vivadent, Schaan, Liechtenstein) (group 2), Gradia Core self-etching bond/Gradia Core cement (GC, Tokyo, Japan) (group 3). All products were handled according to the manufacturer's instructions. The batch numbers, adhesive strategy, curing method, application mode of the materials used in the study are reported in Table 1 . Light curing was performed using a LED light $\left(1200 \mathrm{~mW} / \mathrm{cm}^{2}\right.$ output; B.A. Optima 10 ; B.A. International Ltd., Northampton, UK). The exposed dentin along the coronal part of the root of all prepared specimens was sealed with flowable composite (G-aenial Bond/G-aenial Flo; GC America Inc., Alsip, IL, USA). All the post-cemented roots were placed in water at room temperature for $2 \mathrm{~h}$ until further use.

\section{Preparation of roots for push-out bond strength testing}

Five sections of $1.00 \pm 0.05 \mathrm{~mm}$ were obtained per specimen ( 150 sections in total) using a water-cooled diamond blade (Isomet; Buehler, Lake Bluff, IL, USA). The actual thickness of each slice was measured with a digital calliper (Orteam, Milan Italy) with an accuracy of $0.01 \mathrm{~mm}$.

Each slice was marked on its coronal side with an indelible marker and attached to a horizontal stainless steel platform with an adhesive mounting tape (Scotch; 3M, St. Paul, MN, USA). Loading was performed on a universal testing machine (Triax Digital 50; Controls, Milan, Italy) at a speed of $0.5 \mathrm{~mm} /$ minute until bond failure occurred. Each slice was loaded with a $1 \mathrm{~mm}$ diameter cylindrical plunger tip for the cervical and middle thirds and with a $0.7 \mathrm{~mm}$ diameter cylindrical plunger for the apical thirds that provided the maximum coverage over the post without touching the canal wall. The load was applied to the apical aspect of the root slice and in an apical-coronal direction, so as to push the post toward the larger part of the root slice, thus avoiding any limitation to the material movement. Bond failure load was noted when a sharp decline was observed on the graph and/or complete dislodgement of the post.

To express the bond strength in megapascals (MPa), 
Table 1. Batch numbers, adhesive strategy, curing method, application mode of the materials used in the study

\begin{tabular}{|c|c|c|c|c|c|}
\hline Luting agent & Bonding agent & Manufacturer & Adhesive strategy & Curing method & Application mode \\
\hline $\begin{array}{l}\text { MSM-107 Clear, } \\
130208\end{array}$ & $\begin{array}{l}\text { Self etching primer } \\
\text { A, A130208 } \\
\text { Self etching primer } \\
\text { B, B130208 }\end{array}$ & GC, Tokyo, Japan & Etch-and-rinse & Dual-curing & $\begin{array}{l}\text { Etching of the root canal with } \\
\text { phosphoric acid for } 15 \mathrm{~s} \text {. } \\
\text { Mixing self etching primer } \\
\text { A and B for } 5 \mathrm{~s} \text {. Applying to the } \\
\text { post space and waiting for } 30 \\
\mathrm{~s} \text {. Drying with medium air } \\
\text { pressure for } 10 \mathrm{~s} \text {. Dispensing } \\
\text { resin cement into the post } \\
\text { space. Seating the post into } \\
\text { the root canal. Light curing for } \\
40 \mathrm{~s} \text {. }\end{array}$ \\
\hline $\begin{array}{l}\text { Variolink II, shade } \\
\text { transparent } \\
\text { Base, S04129 } \\
\text { Catalyst, S01620 }\end{array}$ & $\begin{array}{l}\text { ExciTE }^{\oplus} \text { F DSC, } \\
\text { R64753 }\end{array}$ & $\begin{array}{l}\text { Ivoclar Vivadent, } \\
\text { Schaan, } \\
\text { Liechtenstein }\end{array}$ & Etch-and-rinse & Dual-curing & $\begin{array}{l}\text { Etching of the root canal with } \\
\text { phosphoric acid for } 15 \mathrm{~s} \text {. } \\
\text { Activation of ExciTE } \text { F DSC. } \\
\text { Applying ExciTE } \text { F DSC for } 10 \\
\text { s. Mixing the base and the } \\
\text { catalyst of Variolink II and } \\
\text { applying the mixed material to } \\
\text { the post. Seating the post into } \\
\text { the root canal. Light curing for } \\
40 \mathrm{~s} \text {. }\end{array}$ \\
\hline $\begin{array}{l}\text { Gradia Core } \\
\text { Dual-cured } \\
\text { radiopaque } \\
\text { composite, } \\
1305071\end{array}$ & $\begin{array}{l}\text { Gradia Core self } \\
\text { etching bond A, } \\
1405031 \\
\text { Gradia Core self } \\
\text { etching bond B, } \\
1405121\end{array}$ & GC, Tokyo, Japan & Self-etch & Dual-curing & $\begin{array}{l}\text { Mixing self etching bond } \mathrm{A} \\
\text { and } \mathrm{B} \text { for } 5 \mathrm{~s} \text {. Applying to the } \\
\text { post space and waiting for } 30 \\
\text { s. Drying with medium air } \\
\text { pressure for } 10 \mathrm{~s} \text {. Light curing } \\
\text { for } 10 \mathrm{~s} \text {. } \\
\text { Dispensing dual-cured luting } \\
\text { cement into the post space. } \\
\text { Seating the post into the root } \\
\text { canal. Light curing for } 20 \mathrm{~s} \text {. }\end{array}$ \\
\hline
\end{tabular}

the load at failure recorded in newtons $(\mathrm{N})$ was divided by the area of the bonded interface (A) as calculated by the following formula $A=\pi\left(r_{1}+r_{2}\right)\left[\left(h^{2}+r_{1}-r_{2}\right)^{2}\right]^{0.5}$, where $\pi$ is the constant $3.14, r_{1}$ is the coronal post radius, $r_{2}$ is the apical post radius and $\mathrm{h}$ is the thickness of the slice in millimeters. All debonded specimens were analyzed using a stereomicroscope (Nikon SMZ645; Nikon, Tokyo, Japan) at 40x magnification, and failures were classified as follows: adhesive between the cement and the dentin $(\mathrm{AD})$; adhesive between the cement and the post (AP); fracture of the specimen (cohesive within the post and the dentin $[\mathrm{FR}])$; and mixed failure $(\mathrm{M})$.

\section{Statistical analysis}

A preliminary linear regression analysis was conducted in each group to check if the root of origin was a significant factor for differences in push-out strengths of root slices.
The results revealed that in none of the groups did the root of origin significantly influence the measured bond strength. Therefore, the slices were considered as independent statistical units within each experimental group. The results of tests were expressed as the number of observations $(\mathrm{n})$, mean \pm standard deviation, median and minmax values. The results of the homogenity (Levene's Test) and normality tests (Shapiro Wilk) were used to decide which statistical methods to apply in the comparison of the study groups. Normally distributed and with homogeneous variances groups were compared three or more groups by Analysis of Variance. Multiple comparison tests, the Tukey HSD test was used. According to those tests results parametric test assumptions were not available for some variables, so the comparisons three independent groups were performed by Kruskal Wallis test. Multiple comparison tests, the adjusted Bonferroni test was used. All statistical analyses were performed with the SPSS 
Table 2. Descriptive statistics of the push-out bond strength values. Different letters indicate statistically significant differences among groups $(p<0.05)$

\begin{tabular}{lcccc} 
Group & Sample size & Mean & Standard deviation & Significance $\mathbf{p}<\mathbf{0 . 0 5}$ \\
\hline MSM-107 & 10 & 9.61 & 2.98 & $\mathrm{~A}$ \\
ExciTE ${ }^{\oplus}$ F DSC/Variolink II & 10 & 6.57 & 3.54 & $\mathrm{AB}$ \\
Gradia Core & 10 & 4.79 & 2.47 & $\mathrm{~B}$ \\
\hline
\end{tabular}

Table 3. Failure mode distribution

\begin{tabular}{lcccc} 
Group & \multicolumn{4}{c}{ Failure mode (n) } \\
\cline { 2 - 5 } & AD & AP & FR & M \\
MSM-107 & 9 & 15 & 10 & 16 \\
ExciTE ${ }^{\oplus}$ F DSC/Variolink II & 3 & 17 & 0 & 30 \\
Gradia Core & 12 & 12 & 0 & 26 \\
\hline
\end{tabular}

AD: Adhesive between the cement and the dentin; AP: Adhesive between the cement and the post; FR: Fracture of the specimen (cohesive within the post and the dentin); $M$, and mixed failure.

software (SPSS Ver. 17.0; SPSS Inc., Chicago IL, USA). $P$ value of $<.05$ was considered statistically significant.

\section{Results}

The push-out bond strengths of resin cements are shown in Table 2. There were significant differences in bond strength amongst groups. Experimental resin cement MSM-107 in combination with etch and rinse application (group 1) achieved the highest bond strength $(9.61 \pm 2.98)$. The mean value recorded in group 3 (Gradia Core $)(4.79 \pm 2.47)$ were significantly lower than group $\mathrm{l}(\mathrm{p}<0.05)$ and did not differ from group $2\left(\operatorname{ExciTE}^{\oplus} \mathrm{F}\right.$ DSC/Variolink II) $(6.57 \pm 3.54)(\mathrm{p}>0.05)$. There was also no significant difference between groups 1 and $2(p>0.05)$.

\section{Failure modes}

Stereomicroscopic examination of 150 samples revealed that the bond failure to be mainly mixed failure in $\mathrm{Ex}$ ciTE $^{\circledR}$ F DSC/Variolink II and Gradia-Core groups. Adhesive failures between the cement and the post, cohesive failures within the post and the dentin were mostly observed in MSM-107 group (Table 3).

\section{Discussion}

Bond-strength testing has become a popular method for determining the effectiveness of adhesion between endodontic materials and tooth structure. Roydhouse ${ }^{[12]}$ introduce extrusion testing in operative dentistry with a system in which composite cylinders were pushed out from dentin disks. ${ }^{[13]}$ The extrusion design generates polymerization stresses that similar to those that occur in clinical situations. ${ }^{[14]}$ Microtensile pull-out and push-out tests have been traditionally used to assess the retention of posts in the root canal. ${ }^{[15,16]}$ The push-out test is based on shear stress at the interface between dentin and luting material. ${ }^{[17]}$ In the present study, the push-out test was performed 24 hours after adhesive cementation procedures because bond strength can increase during this period. ${ }^{[18]}$ On the other hand, no aging or rather simulation of clinical function, i.e. thermocycling, cyclic mechanical loading or long-term storage of the specimens was planned for the present study. This short interval fails to provide information about the long-term performance of the materials. It could be interesting to investigate the long-term behavior of the bond following storage in a stimulated oral environment, possibly in combination with thermocycling and/or mechanical loading, in order to mimic clinical function.

In the light of the push-out test results and the analysis of dentin interfaces, the null hypothesis, that there are no differences in bond strengths amongst the luting systems, was rejected. All three of the groups tested showed measurable adhesive properties. The new experimental etch \& rinse resin system MSM-107 had the highest bond strength, whereas the Gradia Core self-etching bond/ Gradia Core cement combination had the lowest values.

The reported bond strengths of etch and rinse adhesives to root canal compared to self-etch adhesives are inconsistent and seem to be dependent on the luting material used. ${ }^{[16,19,20]}$ Nevertheless, it was asumed that combining all the fiber post system components (adhesive, resin cement, and fiber post) from the same manufacturer would prevent possible incompatibility between the materials and allow assessment of the full potential of each system under laboratory conditions. ${ }^{[20]}$ The only exception was made in the case of ExciTE ${ }^{\circledR}$ F DSC adhesive/Variolink II group. This may partly serve an explanation for the insignificant difference in bond strength between ExciTE $^{\circledR} \mathrm{F}$ DSC adhesive/Variolink II group and GradiaCore group, as it was previously reported that push-out strength may be influenced by a type of fiber post to a greater extent than by a luting agent. ${ }^{[21]}$

A current trend in dental marketing is the use of dualcure post luting cements that may also act as core build-up materials, due to their high filler content, which increases 
their physical properties. Dual-cured cement, investigated in group 3, is a material formulated to be used for both post cementation and the core build-up procedure. A recent study reported that when resin cements with $10 \mathrm{wt} \%$ or $30 \mathrm{wt} \%$ filler load were used to lute fiber posts, higher push-out strengths and lower interfacial nanoleakage were recorded, in comparison with $50 \mathrm{wt} \%$ or $70 \mathrm{wt} \%$ filled cement. ${ }^{[22]}$ Therefore it can be speculated that the high content of filler $(70-75 \%)^{[23]}$ may have negatively affected the bond strength of Gradia-Core. Furthermore, self-etch adhesives include acidic monomer solutions either making the smear layer permeable to allow a formation of hybrid layer interface or hybridized smear layer. The penetrating ability of the dentin smear layer is dependent to smear layer thickness and aggressiveness of self-etch monomers. ${ }^{[24]}$ When the self-etch monomer does not etch profoundly enough, the adhesive will be unable to establish a firm bond with the intact dentin ${ }^{[19]}$ Retention and 4-year survival rate of fiber posts cemented with Gradia-Core system was comparable with the results obtained with self-adhesive cements. ${ }^{[25-27]}$ However, comparative studies between etch and rinse and Gradia-Core system is currently lacking in the literature.

Scarce information is also available on the resin cement MSM-107. The results of the present study are corroborated by other investigators, who evaluated the wear resistances of several resin cements using the three body wear test and obtained significantly higher wear resistance for the MSM-107, compared to self-etch adhesives and Variolink II. ${ }^{[28]}$

Although promising results for the use of fiber posts have been reported, ${ }^{[29]}$ problems associated with cementation, bond integrity, and interface stability have not been entirely solved. ${ }^{[30]}$ The bond strength between the fiber post and the radicular dentin was affected by the curing mode, the working time, the mixing method, the filler contents, the flow, the composition of the resin matrix, and the moisture control of the root canal. The degree of conversion of the resin cements, the shape of the root canal, the position of post in the root canal, and the space occupied by the resin cement between post and the radicular dentin are also influential factors that require further study. ${ }^{[31]}$

\section{Conclusion}

Within the limitations of the present study, it may concluded that the push-out bond strength of fiber posts was significantly influenced by luting agents. In the test arrangement used, the self-etching approach may offer less favourable adhesion to root canal dentin in comparison with newly developed etch and rinse approach, MSM-107.

\section{Acknowledgement}

The author would like to thank Prof. Marco Ferrari, Prof. Cecilia Goracci and Prof. Simone Grandini for the materials and research facilities they provided.

Conflict of interest: None declared.

\section{References}

1. Goldberg AJ, Burstone CJ. The use of continuous fiber reinforcement in dentistry. Dent Mater 1992;8:197-202.

2. Goracci C, Ferrari M. Current perspectives on post systems: a literature review. Aust Dent J 2011;56 Suppl 1:7783. Crosstef

3. Asmussen E, Peutzfeldt A, Heitmann T. Stiffness, elastic limit, and strength of newer types of endodontic posts. J Dent 1999;27:275-8, Crossef

4. Dietschi D, Duc O, Krejci I, Sadan A. Biomechanical considerations for the restoration of endodontically treated teeth: a systematic review of the literature, Part II (Evaluation of fatigue behavior, interfaces, and in vivo studies). Quintessence Int 2008;39:117-29.

5. Cheung W. A review of the management of endodontically treated teeth. Post, core and the final restoration. J Am Dent Assoc 2005;136:611-9. Crossref

6. Van Meerbeek B, De Munck J, Yoshida Y, Inoue S, Vargas $\mathrm{M}$, Vijay $\mathrm{P}$, et al. Buonocore memorial lecture. Adhesion to enamel and dentin: current status and future challenges. Oper Dent 2003;28:215-35.

7. Perdigão J, Geraldeli S. Bonding characteristics of selfetching adhesives to intact versus prepared enamel. J Esthet Restor Dent 2003;15:32-42. Crossref

8. Senawongse P, Harnirattisai C, Shimada Y, Tagami J. Effective bond strength of current adhesive systems on deciduous and permanent dentin. Oper Dent 2004;29:196202.

9. Shimada Y, Senawongse P, Harnirattisai C, Burrow MF, Nakaoki Y, Tagami J. Bond strength of two adhesive systems to primary and permanent enamel. Oper Dent 2002;27:403-9.

10. Sardella TN, de Castro FL, Sanabe ME, Hebling J. Shortening of primary dentin etching time and its implication on bond strength. J Dent 2005;33:355-62. Crossref

11. Bitter K, Paris S, Martus P, Schartner R, Kielbassa AM. A Confocal Laser Scanning Microscope investigation of different dental adhesives bonded to root canal dentine. Int Endod J 2004;37:840-8. Crosssef

12. Roydhouse RH. Punch-shear test for dental purposes. J Dent Res 1970;49:131-6. Crossref

13. Watanabe I, Nakabayashi N. Measurement methods for adhesion to dentine: the current status in Japan. J Dent 1994;22:67-72. Crossref

14. Frankenberger R, Krämer $\mathrm{N}$, Oberschachtsiek $\mathrm{H}$, 
Petschelt A. Dentin bond strength and marginal adaption after $\mathrm{NaOCl}$ pre-treatment. Oper Dent 2000;25:40-5.

15. Goracci C, Grandini S, Bossù M, Bertelli E, Ferrari M. Laboratory assessment of the retentive potential of adhesive posts: a review. J Dent 2007;35:827-35. Crossref

16. Onay EO, Korkmaz Y, Kiremitci A. Effect of adhesive system type and root region on the push-out bond strength of glass-fibre posts to radicular dentine. Int Endod J 2010;43:259-68. Crossref

17. Goracci C, Raffaelli O, Monticelli F, Balleri B, Bertelli E, Ferrari $M$. The adhesion between prefabricated FRC posts and composite resin cores: microtensile bond strength with and without post-silanization. Dent Mater 2005;21:43744. Crossref

18. Sadek FT, Goracci C, Monticelli F, Grandini S, Cury AH, Tay F, et al. Immediate and 24-hour evaluation of the interfacial strengths of fiber posts. J Endod 2006;32:1174-7.

19. Sterzenbach G, Karajouli G, Naumann M, Peroz I, Bitter K. Fiber post placement with core build-up materials or resin cements-an evaluation of different adhesive approaches. Acta Odontol Scand 2012;70:368-76. Crossref

20. Radovic I, Mazzitelli C, Chieffi N, Ferrari M. Evaluation of the adhesion of fiber posts cemented using different adhesive approaches. Eur J Oral Sci 2008;116:557-63. Crossref

21. Kurtz JS, Perdigão J, Geraldeli S, Hodges JS, Bowles WR. Bond strengths of tooth-colored posts, effect of sealer, dentin adhesive, and root region. Am J Dent 2003;16:31-6.

22. Ferrari M, Carvalho CA, Goracci C, Antoniolli F, Mazzoni A, Mazzotti G, et al. Influence of luting material filler content on post cementation. J Dent Res 2009;88:951-6.

23. Ding H, Meng X, Luo X. Hardness gradients of dualpolymerized flowable composite resins in simulated root canals. J Prosthet Dent 2014;112:1231-7. Crossref

24. Tay FR, Pashley DH. Aggressiveness of contemporary self-etching systems. I: Depth of penetration beyond dentin smear layers. Dent Mater 2001;17:296-308. Crossref

25. Juloski J, Fadda GM, Radovic I, Chieffi N, Vulicevic ZR, Aragoneses JM, et al. Push-out bond strength of an experimental self-adhesive resin cement. Eur J Oral Sci 2013;121:50-6. Crossref

26. Coniglio I, Magni E, Cantoro A, Goracci C, Ferrari M. Push-out bond strength of circular and oval-shaped fiber posts. Clin Oral Investig 2011;15:667-72. Crossref

27. Juloski J, Fadda GM, Monticelli F, Fajó-Pascual M, Goracci C, Ferrari M. Four-year Survival of Endodontically Treated Premolars Restored with Fiber Posts. J Dent Res 2014;93(7 Suppl):52-8, Crossref

28. Matsumoto M, Arita A, Ikushima K, Kumagai T, Sakuma T. Wear resistance of a new resin-cement, MSM-107. Proceedings of the 6th General Session of the Pan European Region of the International Association for Dental Research; Abstract \#132. Helsinki, Finland: International Association for Dental Research 2012.

29. Monticelli F, Grandini S, Goracci C, Ferrari M. Clinical behavior of translucent-fiber posts: a 2-year prospective study. Int J Prosthodont 2003;16:593-6.

30. Bonfante EA, Pegoraro LF, de Góes MF, Carvalho RM. SEM observation of the bond integrity of fiber-reinforced composite posts cemented into root canals. Dent Mater 2008;24:483-91. Crossref

31. Chang HS, Noh YS, Lee Y, Min KS, Bae JM. Push-out bond strengths of fiber-reinforced composite posts with various resin cements according to the root level. J Adv Prosthodont 2013;5:278-86. Crossref 